\title{
A Through-the-Wall Radar Imaging Method Based on a Realistic Model
}

\author{
Tian Jin $^{1,2,3}$ and Alexander Yarovoy ${ }^{2}$ \\ ${ }^{1}$ College of Electronic Science and Engineering, National University of Defense Technology, Changsha 410073, China \\ ${ }^{2}$ Microwave Sensing, Signals and Systems (M3S), TU Delft, 2628 CD Delft, Netherlands \\ ${ }^{3}$ Collaborative Innovation Center of Information Sensing and Understanding, Xi'an, Shaanxi 710071, China
}

Correspondence should be addressed to Tian Jin; tianjin@nudt.edu.cn

Received 19 January 2015; Revised 3 June 2015; Accepted 23 June 2015

Academic Editor: Amelie Litman

Copyright (C) 2015 T. Jin and A. Yarovoy. This is an open access article distributed under the Creative Commons Attribution License, which permits unrestricted use, distribution, and reproduction in any medium, provided the original work is properly cited.

An image focusing method based on a realistic model for a wall is proposed for through-the-wall radar imaging using a multipleinput multiple-output array. A technique to estimate the wall parameters (i.e., position, thickness, and permittivity) from the radar returns is developed and tested. The estimated wall properties are used in the developed penetrating image formation to form images. The penetrating image formation developed is computationally efficient to realize real-time imaging, which does not depend on refraction points. The through-the-wall imaging method is validated on simulated and real data. It is shown that the proposed method provides high localization accuracy of targets concealed behind walls.

\section{Introduction}

Through-the-wall imaging (TWI) and, in general, imaging of buildings internal structure have received much interest in recent years [1-4]. The crucial point in TWI is the presence of the wall. Traditional image formations are mostly based on the assumption of homogenous propagation medium, which is no longer valid in TWI. Refraction of electromagnetic (EM) wave may occur at the front and rear surfaces of the wall, where the propagation path is not a straight line any more. Although the traditional image formations, that is, the back-projection (BP) algorithm, still work if the influence of the wall is not taken into account, the formed image of behind-the-wall targets will be blurred and displaced from their true locations, which degrades radar performance.

In order to obtain a good focusing quality of behind-thewall targets, the effect of wall should be compensated during the imaging procedure, where the wall parameters need to be known or estimated. Fortunately, radar receives backscattered signals from targets of interest and their surroundings, that is, the wall, which potentially allows extracting the surrounding information from the received echo.

In TWI, the wall parameters, such as width and permittivity, are required for good quality imaging. There are some methods to estimate the wall parameters, such as using different array structures or multiple standoff distances [5], but they involve additional measurement procedures. Some approaches use iterative optimization schemes [6], but they are computationally intensive. Other authors retrieved the unknown wall parameters by using an inverse scattering algorithm, which minimizes the mean square error between the measured and the predicted reflection coefficient calculated from a forward model [7]. The inverse approaches require precise measurement with a high dynamic range and a time-consuming calibration procedure. The permittivity of a wall is commonly estimated from the amplitude of the echoes backscattered from the front surface of the wall [8]. However, this approach requires precise calibration similar to inverse methods. Therefore, estimation of wall parameters using radar return needs further study in TWI. There are several through-the-wall image formations that can be used in TWI [9-12], where the refraction point needs to be determined to calculate the propagation path between the transmitting (and/or receiving) antenna and the behindthe-wall target. A precise but time-consuming method is proposed to calculate the refraction point by solving several goniometrical functions [9]. In order to simplify the computation, an equivalent two-layer model is introduced 


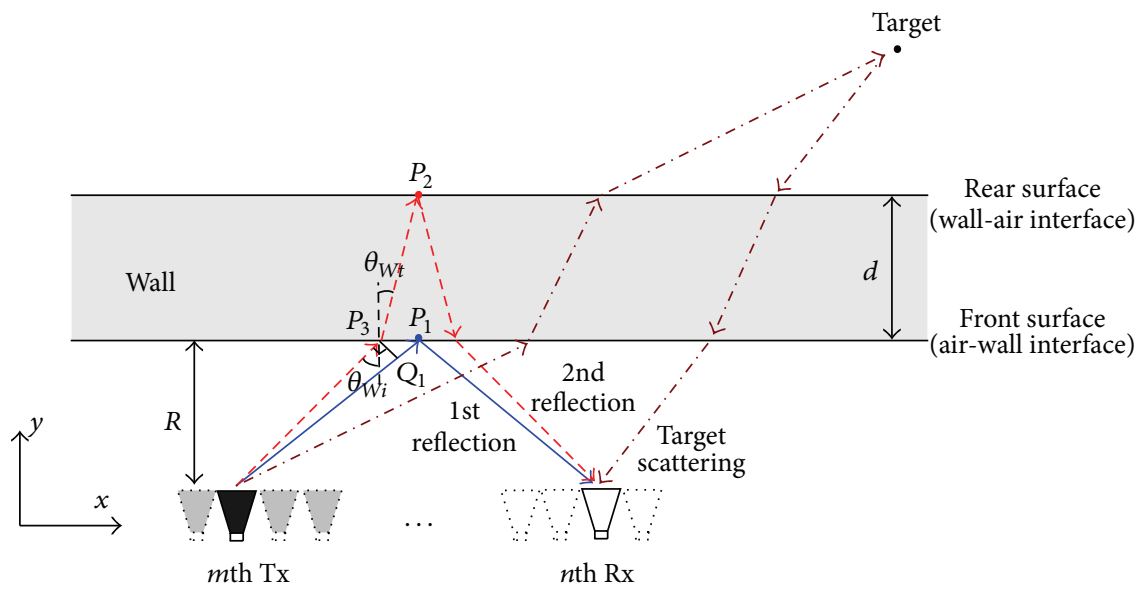

FIGURE 1: Imaging geometry of TWR with MIMO array.

to represent the three-layer model (air-wall-air) without introducing any error in calculation of propagation path, where only one refraction point needs to be determined [13]. However, the position of the refraction point changes with the positions of transmitting/receiving antennas. Therefore, the calculation of refraction point has to be done for each pair of transmitting/receiving antennas with respect to every imaging grid point, which is still a huge computational burden even with the equivalent two-layer model. In order to reduce the computational complexity, a novel penetrating image formation is proposed, which does not need to determine the refraction point and thus is referred to as refraction-point-free (RPF) penetrating image formation.

In this paper, we propose a novel through-the-wall image formation for virtual aperture radar (VAR). Compared with synthetic aperture radar (SAR), which obtains the imaging aperture by the platform movement, a VAR system forms the imaging aperture by a multiple-input multiple-output (MIMO) array. For a MIMO array with $M$ transmitting antennas and $N$ receiving antennas, a virtual aperture with $M \times N$ virtual transceivers can be obtained. The data acquisition time of a virtual aperture is usually in the order of $m s$, which is much shorter than that of a synthetic aperture. Therefore, a moving target, defocusing in SAR images, can be well focused in VAR images because of the fact that a moving target, that is, a moving person, can be considered stationary during such a short time. Therefore, VAR systems are widely adopted in TWI. This paper is organized as follows. In Section 2, the realistic model for through-the-wall VAR is developed, which includes three wall parameters: position, width, and permittivity. In Section 3, a method to estimate the three parameters using the radar return is proposed. Based on the estimated wall parameters, the RPF penetrating image formation is proposed to form through-the-wall images. In Section 4, simulated and real data are used to validate the efficiency of our proposed method. Discussions and conclusions are given in Section 5 .

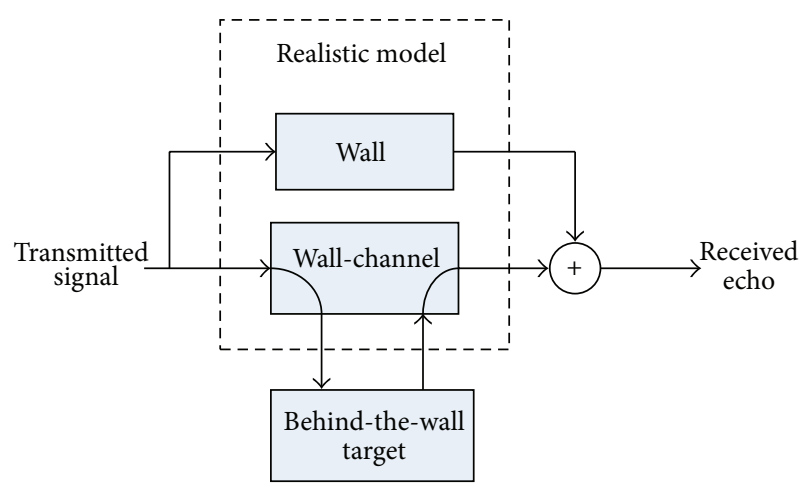

FIgURE 2: Developed realistic model of TWR.

\section{Realistic Model for Through-the-Wall VAR}

In a typical scenario for through-the-wall radar (TWR), a wall separates radar and targets. Figure 1 illustrates the $2 \mathrm{D}$ imaging geometry of a linear MIMO array parallel to the wall. Using the linear MIMO array, we can obtain a linear virtual aperture to form the image in the $x-y$ imaging plane, where $x$ and $y$ denote the cross-range and the down-range, respectively.

The wall and behind-the-wall targets will both cause backscattered signals, where the propagation path of EM wave with respect to the $m$ th transmitting (Tx) and the $n$th receiving $(\mathrm{Rx})$ antennas is depicted in Figure 1. Therefore, the received echo of TWR is composed of two parts: one is from the wall and the other is from behind-the-wall targets. The wall also serves as the propagation channel for the echo model of behind-the-wall targets, as depicted in Figure 2. In TWR imaging, the realistic model is characterized by three wall parameters: position, width, and permittivity. When the linear MIMO array is parallel to the wall, the wall position can be defined by the distance between the wall's front surface and the linear MIMO array.

For the echo from the wall, the transmitted signal will be reflected at the front and rear surfaces, which is denoted as 
the 1st and 2nd reflections, respectively. The echo phase history of the 1st and 2nd reflections can be defined by the electrical length of the two-way path traveled by a spherical wave from the transmitting antenna to the reflection point and back to the receiving antenna.

Given the $m$ th transmitting element and the $n$th receiving element located at $\left(x_{T m}, 0\right)$ and $\left(x_{R n}, 0\right)$, respectively, the propagation path $\ell_{W 1}$ of the 1 st reflection is

$$
\ell_{W 1}=2 r_{T_{X} P_{1}}
$$

where $r_{T_{X} P_{1}}$ is the distance from the transmitting antenna and the reflection point at the front surface $P_{1}$.

The propagation medium of the 2 nd reflection is air-wall, which refracts at $P_{3}$ on the front surface and reflects at $P_{2}$ on the rear surface, whose equivalent propagation path $\ell_{W 2}$ in air is

$$
\ell_{W 2}=2\left(r_{T_{X} P_{3}}+\sqrt{\varepsilon_{r}} r_{P_{3} P_{2}}\right),
$$

where $\varepsilon_{r}$ is the relative permittivity of the wall and $r_{T_{X} P_{3}}$ and $r_{P_{3} P_{2}}$ are the distances from the transmitting antenna to $P_{3}$ and from $P_{3}$ to $P_{2}$, respectively.

When the transmitting signal refracts at the point $P_{3}$, the incident angle $\theta_{W i}$ and the refraction angle $\theta_{W t}$ obey Snell's law as

$$
\frac{\sin \theta_{W i}}{\sin \theta_{W t}}=\sqrt{\varepsilon_{r}} .
$$

According to the geometry relationship depicted in Figure 1, we have

$$
\begin{aligned}
& r_{T_{X} P_{3}} \approx r_{T_{X} P_{1}}-d \tan \theta_{W t} \sin \theta_{W i}, \\
& r_{P_{3} P_{2}}=\frac{d}{\cos \theta_{W t}},
\end{aligned}
$$

where $d$ is the wall width.

Substituting (4) into (2) and considering the relationship of $\theta_{W i}$ and $\theta_{W t}$ shown in (3), one derives

$$
\begin{aligned}
\ell_{W 2} & =2\left(r_{T_{X} P_{1}}+d \sqrt{\varepsilon_{r}-\sin ^{2} \theta_{W i}}\right) \\
& =\ell_{W 1}+2 d \sqrt{\varepsilon_{r}-\sin ^{2} \theta_{W i}} .
\end{aligned}
$$

\section{Proposed Imaging Method}

3.1. Estimation of Wall Parameters. The realistic model is characterized by three wall parameters: position, width, and permittivity, which should be estimated from the received echo in practice. For the MIMO array, the time delay between the 1 st reflection and the 2 nd reflection with respect to the $m$ th transmitting and the $n$th receiving elements is

$$
t_{d}(m, n)=\frac{2 d \sqrt{\varepsilon_{r}-\sin ^{2} \theta_{W i}(m, n)}}{c}
$$

with

$$
\theta_{W i}(m, n) \approx \arctan \left(\frac{\left|x_{R n}-x_{T m}\right|}{2 R}\right),
$$

where $R$ is the distance between the MIMO array and the wall's front surface and can be estimated as

$$
\widehat{R}=\frac{1}{2 M N} \sum_{m=1}^{M} \sum_{n=1}^{N} \sqrt{c^{2} t_{f}^{2}(m, n)-\left(x_{R n}-x_{T m}\right)^{2}},
$$

where $t_{f}(m, n)$ is the time delay of the 1st reflection with respect to the $m$ th transmitting and the $n$th receiving antennas.

The range resolution $\rho_{r}=c /(2 B)$ is determined by the system bandwidth $B$, where $c$ is the speed of EM wave in the free space. According to the imaging geometry, when the system bandwidth fulfils the condition

$$
B>\frac{c \cos \theta_{W t}}{2 d \sqrt{\varepsilon_{r}}},
$$

the reflections of the wall's front and rear surfaces can be separated in the down-range (or fast-time) domain. The relative permittivity of concrete with no reinforcement is in the range of 5 to 9 under different hydration conditions [14]. Assuming $\varepsilon_{r}=6$ and $d=0.2 \mathrm{~m}$, the condition in (9) gives $B>$ $307 \mathrm{MHz}$ for all refraction angles, which is usually fulfilled for most TWI radars.

Based on (6), (7), and (8), we have

$$
0.25 c^{2} t_{d}^{2}(m, n)=d^{2} \varepsilon_{r}-d^{2} \frac{\left(x_{R n}-x_{T m}\right)^{2}}{\left(x_{R n}-x_{T m}\right)^{2}+4 \widehat{R}^{2}},
$$

which is written in the matrix form as

$$
\mathrm{Ap}=\mathbf{b}
$$

with

$$
\begin{aligned}
& \mathbf{A}=\left[\begin{array}{cc}
1 & -\frac{\left(x_{R 1}-x_{T 1}\right)^{2}}{\left(x_{R 1}-x_{T 1}\right)^{2}+4 \widehat{R}^{2}} \\
1 & -\frac{\left(x_{R 2}-x_{T 1}\right)^{2}}{\left(x_{R 2}-x_{T 1}\right)^{2}+4 \widehat{R}^{2}} \\
\vdots & \vdots \\
1 & -\frac{\left(x_{R N}-x_{T M}\right)^{2}}{\left(x_{R N}-x_{T M}\right)^{2}+4 \widehat{R}^{2}}
\end{array}\right]_{M N \times 2} \\
& \mathbf{p}=\left[\begin{array}{c}
d^{2} \varepsilon_{r} \\
d^{2}
\end{array}\right]_{2 \times 1}=\left[\begin{array}{c}
p_{1} \\
p_{2}
\end{array}\right], \\
& \mathbf{b}=\left[\begin{array}{c}
0.25 c^{2} t_{d}^{2}(1,1) \\
0.25 c^{2} t_{d}^{2}(1,2) \\
\vdots \\
0.25 c^{2} t_{d}^{2}(M, N)
\end{array}\right]_{M N \times 1}
\end{aligned}
$$


The solution of (11) is

$$
\mathbf{p}=\left(\mathbf{A}^{T} \mathbf{A}\right)^{-1} \mathbf{A}^{T} \mathbf{b}
$$

where the superscript $(\cdot)^{T}$ is the transpose operator.

The estimations of the width and the relative permittivity are obtained accordingly as

$$
\begin{aligned}
\widehat{d} & =\sqrt{p_{2}} \\
\widehat{\varepsilon}_{r} & =\frac{p_{1}}{p_{2}} .
\end{aligned}
$$

3.2. RPF Penetrating Image Formation. According to the equivalent two-layer model [13], the equivalent electrical length is independent of the distance between the radar and the air-wall interface. Therefore, the equivalent electrical length can be calculated on the geometry depicted in Figure 3 instead, where the MIMO array is on the front surface and $P_{4}$ is the equivalent refraction point on the rear surface. $\theta_{T i}$ and $\theta_{T t}$ are the incident and the refraction angles, respectively, with the relationship of

$$
\frac{\sin \theta_{T i}}{\sin \theta_{T t}}=\sqrt{\varepsilon_{r}} .
$$

Therefore, the equivalent electrical length from the transmitting element to the target in air can be expressed as

$$
\ell_{T x T a r}=\left(r_{T x P_{4}} \sqrt{\varepsilon_{r}}+r_{P_{4} T a r}\right)
$$

where $r_{\mathrm{TxP}_{4}}$ and $r_{P_{4} \mathrm{Tar}}$ are the distances from the transmitting antenna to the refraction point $P_{4}$ and from the refraction point $P_{4}$ to the target, respectively.

According to the geometry relationship depicted in Figure 5, $r_{\mathrm{TxP}_{4}}$ and $r_{P_{4} \mathrm{Tar}}$ are calculated as

$$
\begin{aligned}
r_{T x P_{4}} & =\frac{d}{\cos \theta_{T t}}, \\
r_{P_{4} T a r} & =r_{T x T a r}-r_{P_{4} Q_{2}}=r_{T x T a r}-\frac{d \cos \left(\theta_{T i}-\theta_{T t}\right)}{\cos \theta_{T t}} \\
& =r_{T x T a r}-d\left(\cos \theta_{T i}+\sin \theta_{T i} \tan \theta_{T t}\right),
\end{aligned}
$$

where $r_{\text {TxTar }}$ is the distance from the transmitting antenna to the target.

Substituting (15), (17) into (16), $\ell_{\text {TxTar }}$ can be rewritten as

$$
\ell_{T x T a r}=r_{T x T a r}+d\left(\sqrt{\varepsilon_{r}-\sin ^{2} \theta_{T i}}-\cos \theta_{T i}\right) .
$$

Similarly, the equivalent electrical length from the target to the receiving element $\ell_{\text {TarRx }}$ in air can be calculated as

$$
\ell_{T a r R x}=r_{T a r R x}+d\left(\sqrt{\varepsilon_{r}-\sin ^{2} \theta_{R i}}-\cos \theta_{R i}\right) .
$$

When the target located at $(x, y)$ is behind the wall, the time delay of its return with respect to the $m$ th transmitting and the $n$th receiving antennas can be estimated as

$$
\begin{aligned}
& t_{T 1}(m, n) \\
& =\frac{\left[\sqrt{\left(x-x_{T m}\right)^{2}+\left(y-y_{T m}\right)^{2}}+\sqrt{\left(x-x_{R n}\right)^{2}+\left(y-y_{R n}\right)^{2}}+\widehat{d}\left(\sqrt{\widehat{\varepsilon}_{r}-\sin ^{2} \theta_{T i}(m, n)}-\cos \theta_{T i}(m, n)+\sqrt{\widehat{\varepsilon}_{r}-\sin ^{2} \theta_{R i}(m, n)}-\cos \theta_{R i}(m, n)\right)\right]}{c}
\end{aligned}
$$

with

$$
\begin{aligned}
& \theta_{T i}(m, n) \approx \arctan \left[\frac{\left(x-x_{T m}\right)}{\left(y-y_{T m}\right)}\right], \\
& \theta_{R i}(m, n) \approx \arctan \left[\frac{\left(x-x_{R n}\right)}{\left(y-y_{R n}\right)}\right] .
\end{aligned}
$$

When the target is in front of the wall, the time delay of its return can be computed as

$$
\begin{aligned}
& t_{T 2}(m, n) \\
& =\frac{\left[\sqrt{\left(x-x_{T m}\right)^{2}+\left(y-y_{T m}\right)^{2}}+\sqrt{\left(x-x_{R n}\right)^{2}+\left(y-y_{R n}\right)^{2}}\right]}{c} .
\end{aligned}
$$

According to (20) and (22), based on the realistic model of TWR, the RPF penetrating image formation is

$$
\begin{aligned}
& I(x, y) \\
& = \begin{cases}\sum_{m=1}^{M} \sum_{n=1}^{N} \int s(t, m, n) \delta\left[t-t_{T 1}(m, n)\right] d t, & y \geq \widehat{R}+0.5 \widehat{d} \\
\sum_{m=1}^{M} \sum_{n=1}^{N} \int s(t, m, n) \delta\left[t-t_{T 2}(m, n)\right] d t, & y<\widehat{R}+0.5 \widehat{d},\end{cases}
\end{aligned}
$$

where $I(x, y)$ is the formed image, $t$ is the fast-time, and $s(t, m, n)$ is the received signal. It is noticed that when the transmitting signal is not the impulse signal but the steppedfrequency signal, $s(t, m, n)$ should be the range compressed signal and a phase compensation term should be involved in (23) to ensure coherent accumulation [15].

\section{Verification}

4.1. Simulation Results. In the simulation, the finitedifference time-domain (FDTD) method is used to simulate 


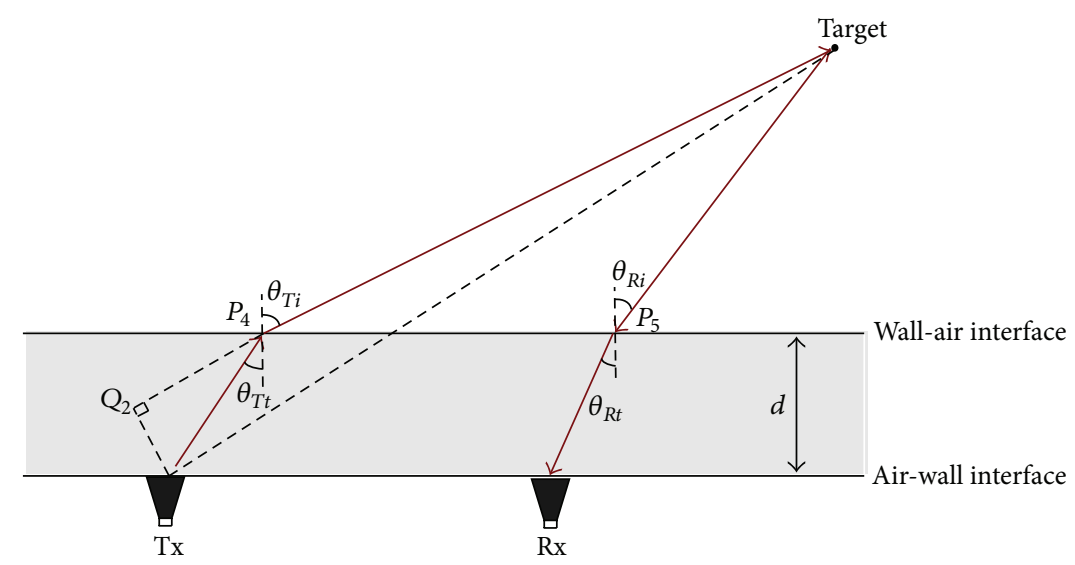

FIGURE 3: Equivalent two-layer propagation model.

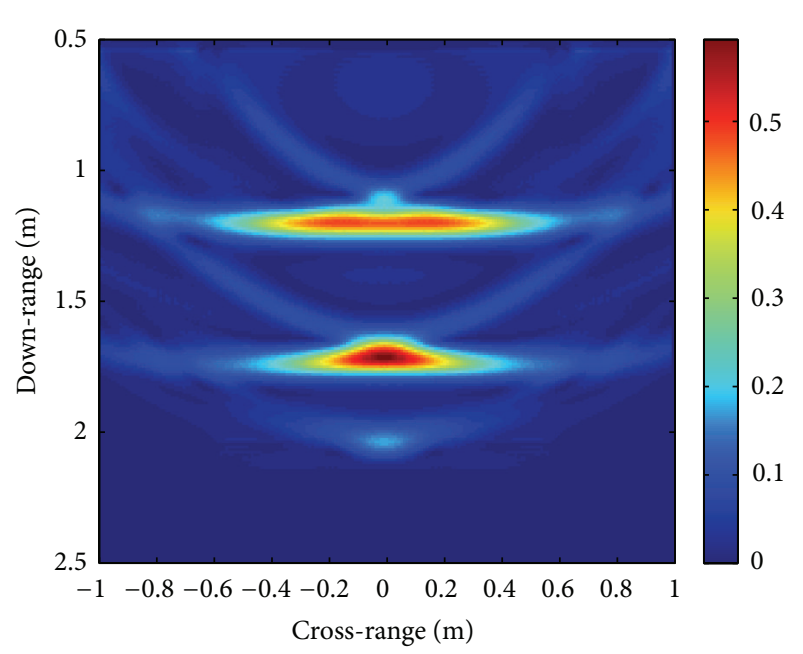

FIGURE 4: Imaging result using traditional imaging method.

the echo. The transmitting signal is the first derivative Gaussian impulse with the lowest and highest frequencies of the transmitted signal being $0.5 \mathrm{GHz}$ and $2 \mathrm{GHz}$, respectively. The antenna array is composed of 1 transmitting antenna and 21 receiving antennas. The receiving antennas form a linear receiving array of $2 \mathrm{~m}$ length. The transmitting antenna is placed at the center of the receiving array with its coordinates $(0,0)$. The array is parallel to the wall with the distance to the front surface of $1.2 \mathrm{~m}$. The thickness of the wall is $0.2 \mathrm{~m}$ and its relative permittivity is 6.25 . One target, with its coordinates of $(0,1.7)$, is behind the wall.

The envelope amplitude image of the $\mathrm{BP}$ imaging result is depicted in Figure 4, where the effect of wall is not considered and thus the image of the target behind the wall is blurred and displaced from its true position with the error of about $0.3 \mathrm{~m}$ in the down-range direction.

The additive white Gaussian noise (AWGN) with zeromean is added to the above FDTD simulated data to evaluate the performance of proposed estimation method under different signal-to-noise ratios (SNRs). The SNR is defined as the power of transmitted signal over the variance of AWGN.

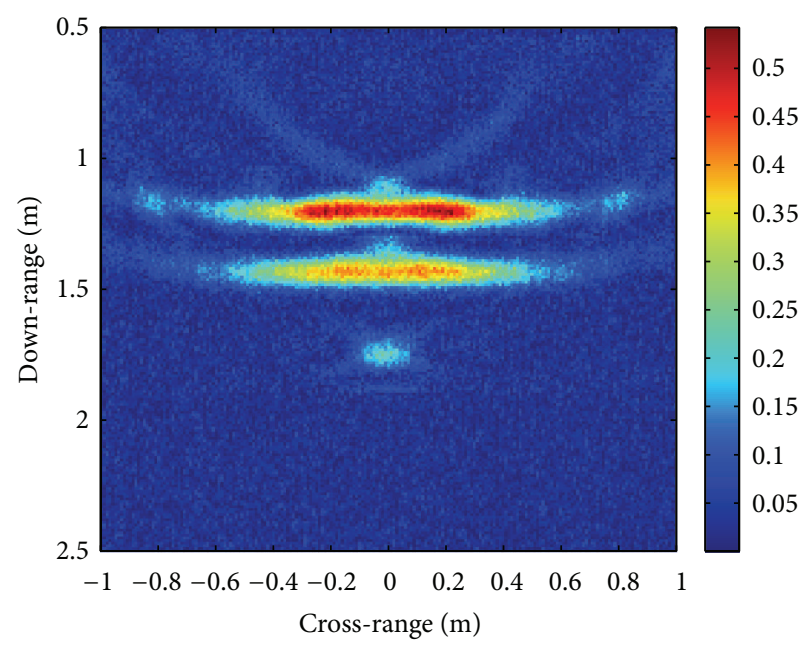

FIGURE 5: Imaging result of proposed method with SNR $=10 \mathrm{~dB}$.

TABLE 1: Mean value of the estimated wall parameters in different SNRs.

\begin{tabular}{lccc}
\hline SNR $(\mathrm{dB})$ & $\widehat{R}(\mathrm{~m})$ & $\widehat{d}(\mathrm{~m})$ & $\widehat{\varepsilon}_{r}$ \\
\hline 5 & 1.19 & 0.220 & 5.355 \\
10 & 1.20 & 0.211 & 6.623 \\
15 & 1.20 & 0.205 & 6.368 \\
20 & 1.20 & 0.203 & 6.291 \\
\hline
\end{tabular}

The estimated wall parameters under different SNRs are shown in Table 1, where 100 Monte Carlo experiments are done for each SNR.

The imaging result of proposed method in $\mathrm{SNR}=10 \mathrm{~dB}$ is depicted in Figure 5. The cross-range resolution is improved from $0.20 \mathrm{~m}$ in Figure 4 to $0.13 \mathrm{~m}$ in Figure 5, and the behindthe-wall target and the rear surface are both corrected to their true positions.

4.2. Real Data Results. We use the real data, collected by our built through-the-wall VAR system (Figure 6), to verify the proposed method further. The VAR system transmits 


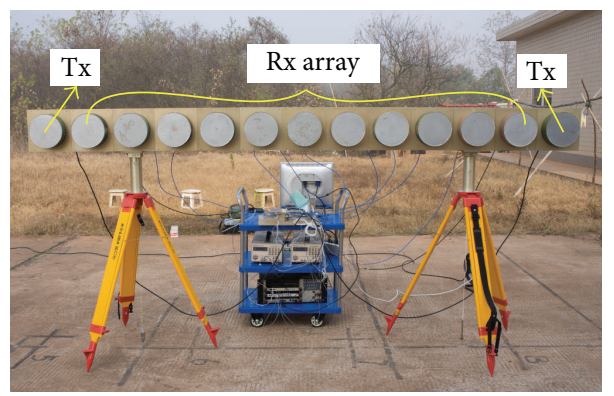

FIGURE 6: Photograph of the through-the-wall VAR system.

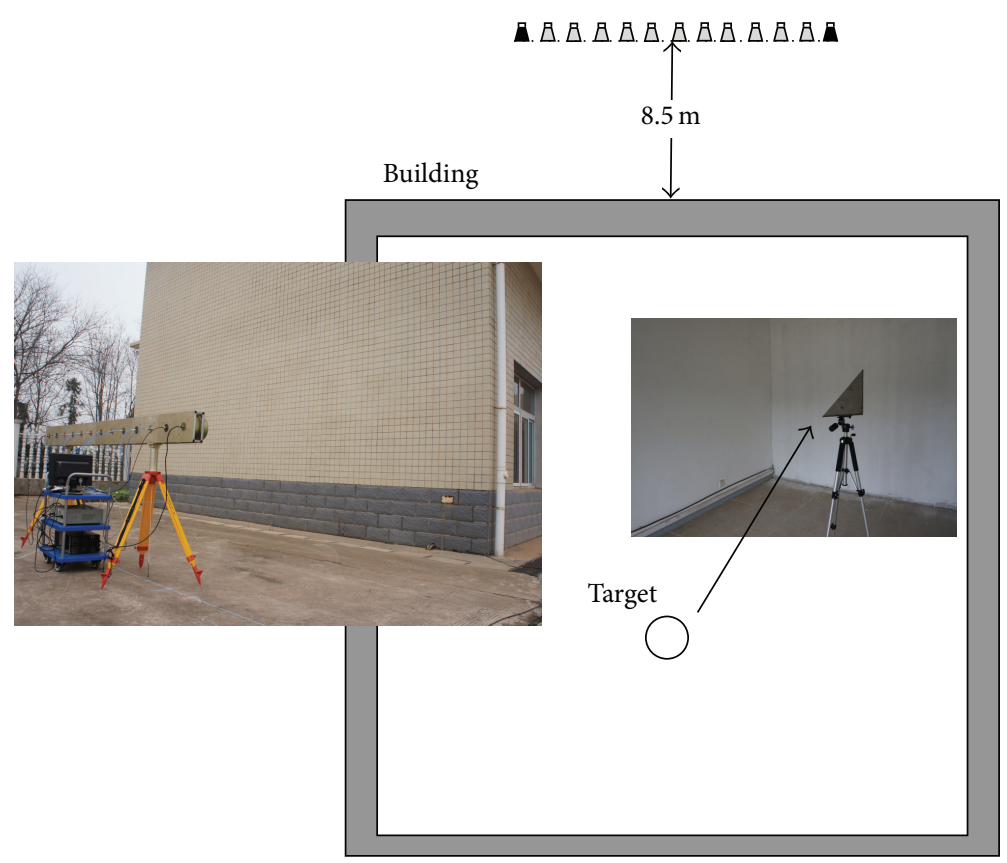

FIgURE 7: Data collection scenario.

the stepped-frequency signal from $500 \mathrm{MHz}$ to $2.5 \mathrm{GHz}$. The MIMO array consists of 2 transmitting and 11 receiving antennas. The receiving antennas, spaced $0.25 \mathrm{~m}$ center to center, form a linear receiving array. The 2 transmitting antennas are located at the two ends of the receiving array and work sequentially. Therefore, there are 22 transmittingreceiving channels in one virtual aperture. The Archimedean spiral antennas are adopted for transmitting and receiving antennas, whose dispersive characteristic should be compensated to ensure the high resolution in down-range. The compensation procedure can be performed via the predistortion technique [16] or the calibration function method [17], where the latter is used in our system.

The experimental scene is depicted in Figure 7. The MIMO array is $8.5 \mathrm{~m}$ away from the exterior wall and a trihedral reflector is in the building. The estimation results of the wall parameters are shown in Table 2.

The imaging results of the BP algorithm and the method proposed are compared in Figure 8, where the dashed circle and the dashed lines indicate the location of the target and the building walls, respectively. If the refraction of
TABLE 2: Estimation of wall parameters for real data.

\begin{tabular}{lccc}
\hline & $R$ & $d$ & $\varepsilon_{r}$ \\
\hline Estimated value & $8.51 \mathrm{~m}$ & $0.27 \mathrm{~m}$ & 5.86 \\
True value & $8.50 \mathrm{~m}$ & $0.28 \mathrm{~m}$ & 5.56 \\
\hline
\end{tabular}

electromagnetic wave is not considered, the locating error is about $0.4 \mathrm{~m}$ in the down-range direction for the target. The result of proposed RPF penetrating image formation with the estimated wall parameters provides almost correct target localization.

\section{Discussions and Conclusions}

In this paper, the realistic model based TWI method is proposed. The way to estimate the wall parameters (i.e., position, width, and permittivity) from the radar return is suggested. A RPF penetrating image formation algorithm is proposed to focus behind-the-wall targets based on the estimated wall parameters, which is computationally efficient to realize the 


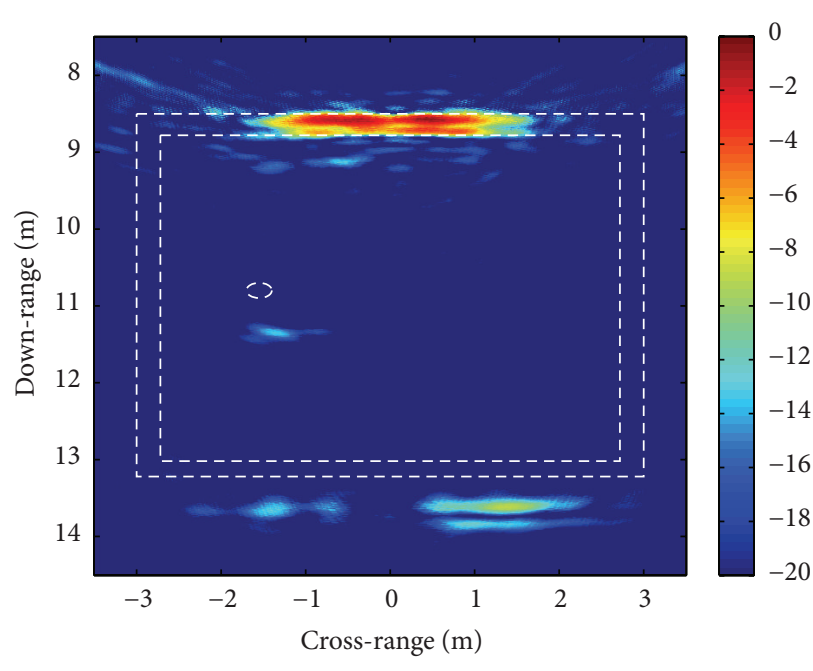

(a)

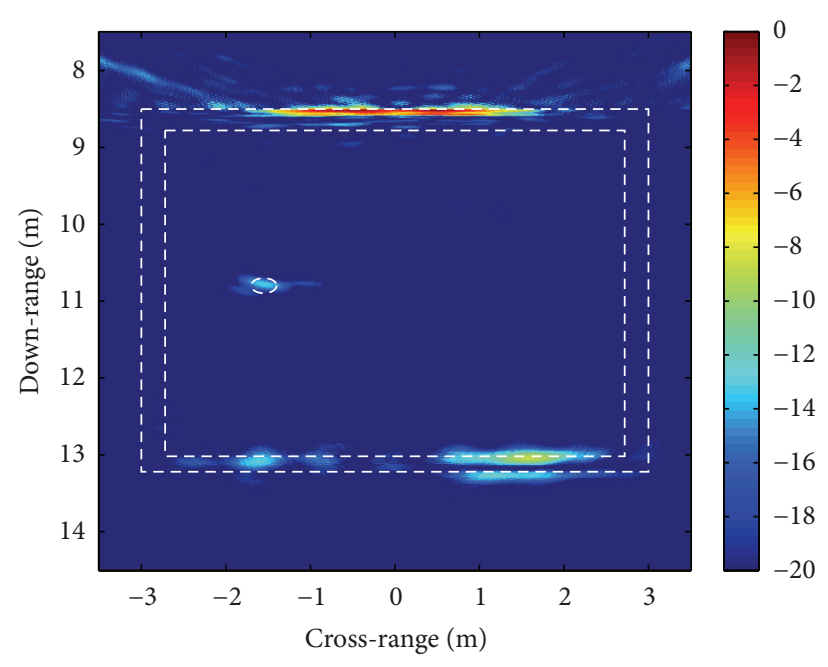

(b)

FIGURE 8: Comparison of imaging results of real data with $20 \mathrm{~dB}$ dynamic range: (a) BP algorithm; (b) proposed method.

real-time imaging. The imaging results of simulated and real data have shown that the proposed method provides high localization accuracy of behind-the-wall targets.

The realistic model with a real-valued permittivity is assumed in this paper. Although the focusing quality can be further improved when considering a more complex realistic model with a frequency-dependent and/or complex-valued permittivity with sacrifice of the computational time, it is not necessary for TWR application in practice. When TWRs are used for detection of behind-the-wall persons, a radar video is more useful than a single radar image in detection, where a computationally efficient imaging method is preferred to increase the frame-rate rather than to obtain the perfect focusing quality. For some walls, their dispersive effect will change the shape of signal and make it hard to discern the 1st and 2 nd reflections from the wall. In such cases, the higher down-range resolution is required. An alternative approach without increasing the hardware burden is to correct the dispersive effect with data processing methods, that is, deconvolution methods. Nevertheless, further research is required.

The proposed realistic model is possible to be extended to describe a multilayered homogenous wall or multiple homogenous walls, whose derivation is analogous to that of the equivalent two-layer model, but more restriction requirement on the down-range resolution is needed. For an inhomogeneous wall, the more complex realistic model is required to characterize the scattering of the wall and its effects on behind-the-wall targets. Fortunately, inhomogeneous walls in practice usually have the periodic structure, such as reinforced concrete walls and cinder block walls, which might simplify the modeling work based on their approximate scattering solutions [18].

\section{Conflict of Interests}

The authors declare that there is no conflict of interests regarding the publication of this paper.

\section{Acknowledgments}

This work was supported by the National Natural Science Foundation of China under Grants 61271441 and 61372161 and the research project of NUDT under Grant CJ12-04-02.

\section{References}

[1] E. J. Baranoski, "Through-wall imaging: historical perspective and future directions," Journal of the Franklin Institute, vol. 345, no. 6, pp. 556-569, 2008.

[2] F. Ahmad, J. Qian, and M. G. Amin, "Wall Clutter mitigation using discrete prolate spheroidal sequences for sparse reconstruction of indoor stationary scenes," IEEE Transactions on Geoscience and Remote Sensing, vol. 53, no. 3, pp. 1549-1557, 2015.

[3] B. Chen, T. Jin, B. Y. Lu, and Z. M. Zhou, "Building interior layout reconstruction from through-the-wall radar image using MST-based method," EURASIP Journal on Advances in Signal Processing, vol. 2014, article no. 31, 2014.

[4] M. Leigsnering, M. Amin, F. Ahmad, and A. M. Zoubir, "Multipath exploitation and suppression for SAR imaging of building interiors: an overview of recent advances," IEEE Signal Processing Magazine, vol. 31, no. 4, pp. 110-119, 2014.

[5] G. Y. Wang and M. G. Amin, "Imaging through unknown walls using different standoff distances," IEEE Transactions on Signal Processing, vol. 54, no. 10, pp. 4015-4025, 2006.

[6] F. Ahmad, M. G. Amin, and G. Mandapati, "Autofocusing of through-the-wall radar imagery under unknown wall characteristics," IEEE Transactions on Image Processing, vol. 16, no. 7, pp. 1785-1795, 2007.

[7] P. C. Chang, R. J. Burkholder, and J. L. Volakis, "Adaptive CLEAN with target refocusing for through-wall image improvement," IEEE Transactions on Antennas and Propagation, vol. 58, no. 1, pp. 155-162, 2010.

[8] M. M. Nikolic, A. Nehorai, and A. R. Djordjevic, "Estimating moving targets behind reinforced walls using radar," IEEE Transactions on Antennas and Propagation, vol. 57, no. 11, pp. 3530-3538, 2009. 
[9] F. Ahmad and M. G. Amin, "A noncoherent approach to through-the-wall radar imaging," in Proceedings of the 8th International Symposium on Signal Processing and its Applications (ISSPA '05), pp. 539-542, August 2005.

[10] P. Withington, H. Fluhler, and S. Nag, "Enhancing homeland security with advanced UWB sensors," IEEE Microwave Magazine, vol. 4, no. 3, pp. 51-58, 2003.

[11] V. Venkatasubramanian and H. Leung, "A novel chaos-based high-resolution imaging technique and its application to through-the-wall imaging," IEEE Signal Processing Letters, vol. 12, no. 7, pp. 528-531, 2005.

[12] F. Ahmad and M. G. Amin, "Noncoherent approach to throughthe-wall radar localization," IEEE Transactions on Aerospace and Electronic Systems, vol. 42, no. 4, pp. 1405-1419, 2006.

[13] T. Jin, B. Chen, and Z. Zhou, "Image-domain estimation of wall parameters for autofocusing of through-the-wall SAR imagery," IEEE Transactions on Geoscience and Remote Sensing, vol. 51, no. 3, pp. 1836-1843, 2013.

[14] C. Thajudeen, A. Hoorfar, F. Ahmad, and T. Dogaru, "Measured complex permittivity of walls with different hydration levels and the effect on power estimation of twri target returns," Progress In Electromagnetics Research B, no. 30, pp. 177-199, 2011.

[15] M. Soumekh, Synthetic Aperture Radar Signal Processing with MATLAB Algorithms, Wiley, 1999.

[16] M. A. Elmansouri and D. S. Filipovic, "Pulse distortion and mitigation thereof in spiral antenna-based UWB communication systems," IEEE Transactions on Antennas and Propagation, vol. 59, no. 10, pp. 3863-3871, 2011.

[17] W. Jian, L. Yanghuan, Z. Zhimin, J. Tian, Y. Yanguang, and W. Yuming, "Image formation techniques for vehicle-mounted forward-looking ground penetrating SAR," in Proceedings of the International Conference on Information and Automation, pp. 667-671, 2008.

[18] M. Dehmollaian and K. Sarabandi, "An approximate solution of scattering from reinforced concrete walls," IEEE Transactions on Antennas and Propagation, vol. 56, no. 8, part 2, pp. 2681-2690, 2008. 

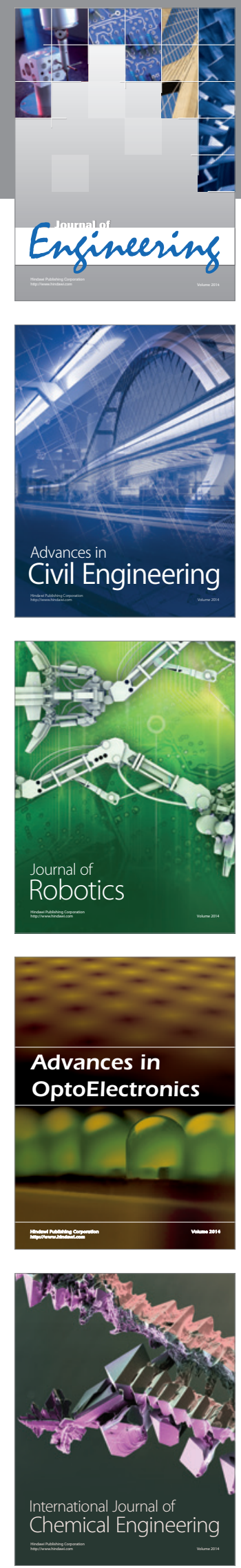

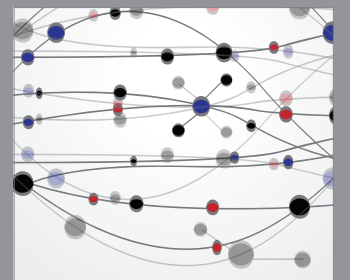

The Scientific World Journal
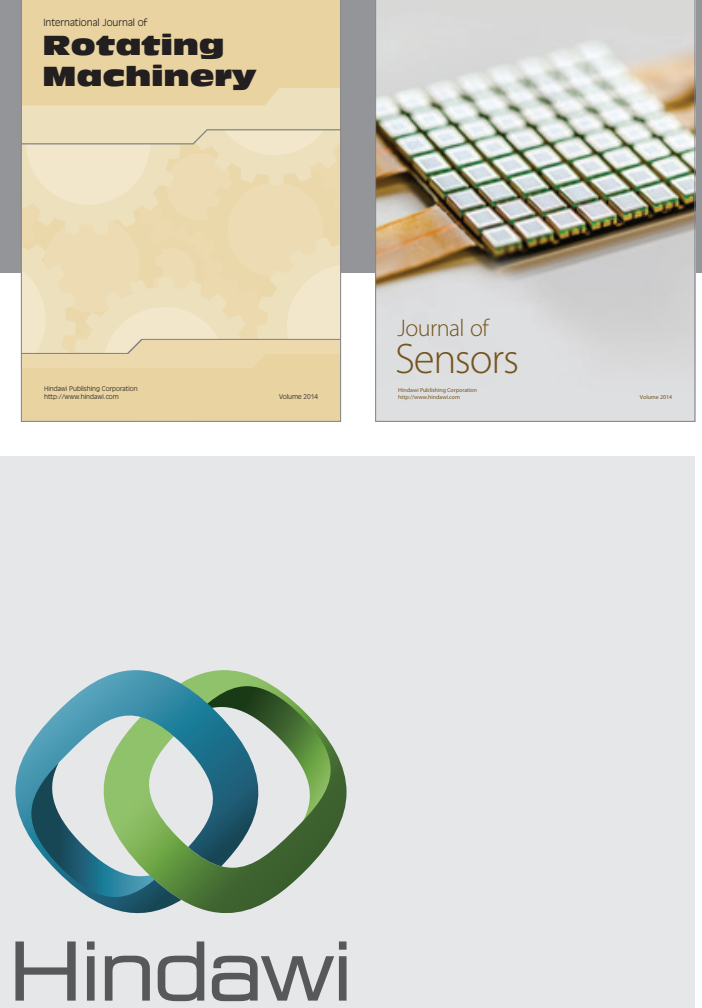

Submit your manuscripts at http://www.hindawi.com
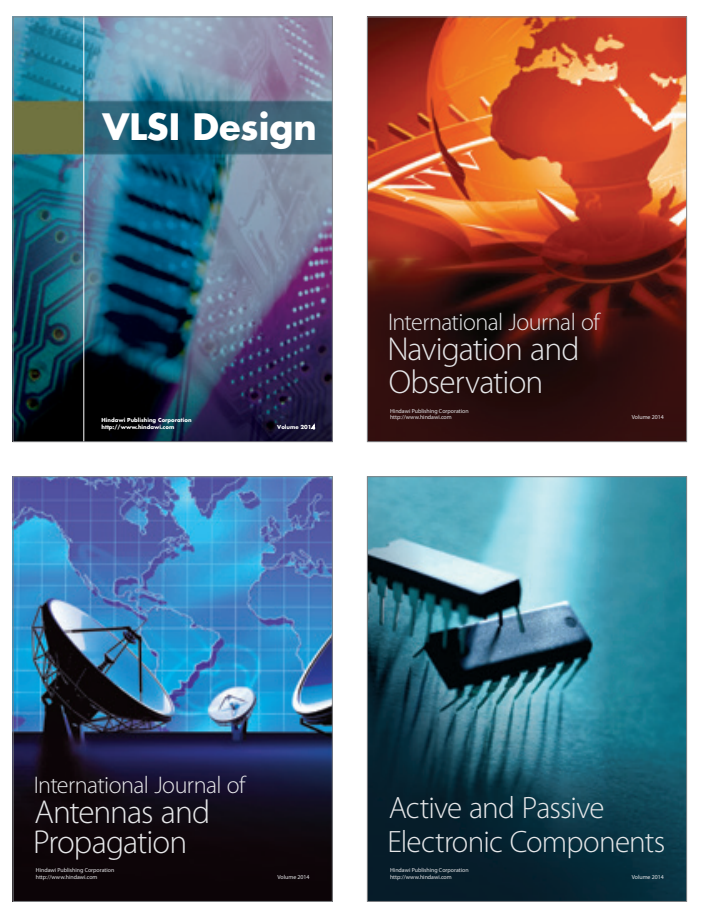
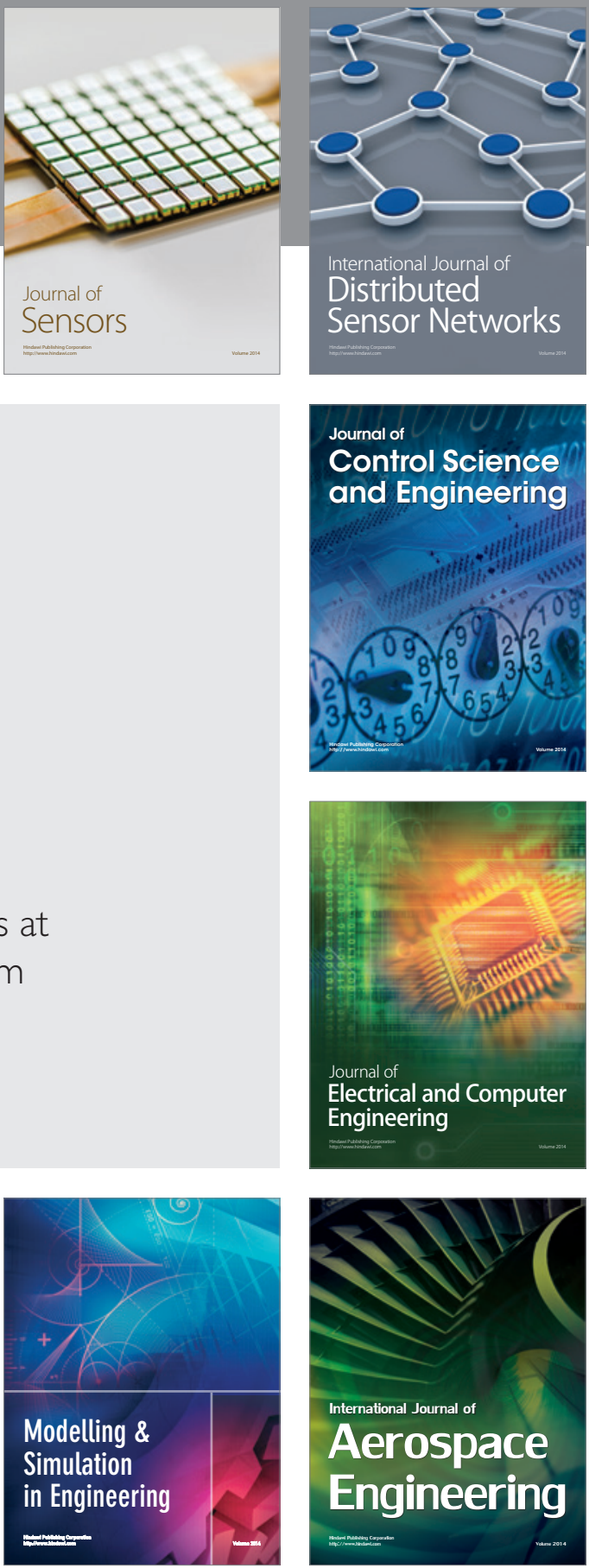

Journal of

Control Science

and Engineering
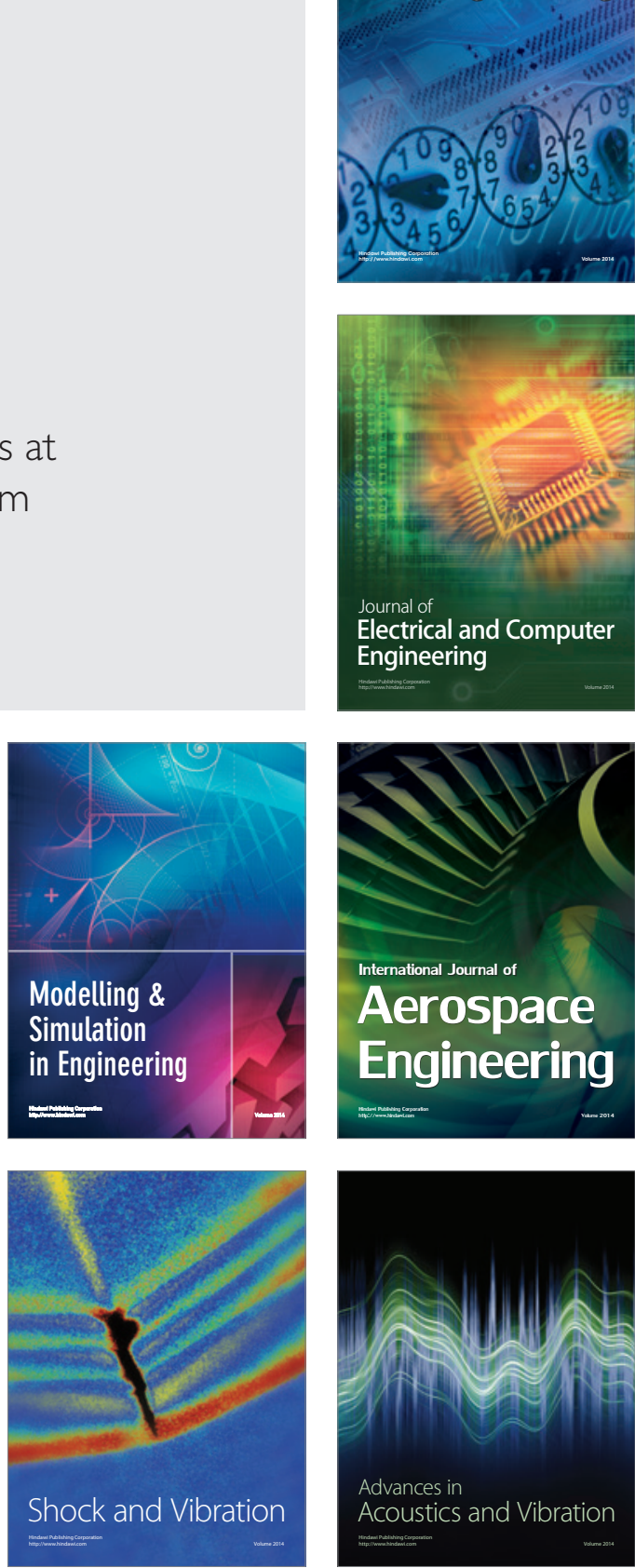